\title{
The development and biological characteristics of a novel potentially radioresistant inbred mouse strain
}

\author{
QIN WANG $^{1}$, LIQING DU $^{1}$, YAN WANG $^{1}, \mathrm{CHANG} \mathrm{XU}^{1}$, ZHIJUAN SUN $^{1}$, YUE FU $^{1}$, BING YANG $^{2}$, \\ YUEYING WANG ${ }^{1}$, CHUANJIE MU $^{1}$, SAIJUN FAN ${ }^{1}$, LU CAI $^{3}$, TAKANORI KATSUBE ${ }^{4}$ and QIANG LIU ${ }^{1}$ \\ ${ }^{1}$ Tianjin Key Laboratory of Radiation Medicine and Molecular Nuclear Medicine, Institute of Radiation Medicine of Chinese \\ Academy of Medical Science, Tianjin 300192; ${ }^{2}$ Department of Cell Biology, Tianjin Medical University, Tianjin 300070, \\ P.R. China; ${ }^{3}$ Department of Pediatrics, University of Louisville, Louisville, KY 40202, USA; ${ }^{4}$ Radiation Risk Reduction \\ Research Program, National Institute of Radiological Sciences, Anagawa 4-9-1, Inage-ku, Chiba 263-8555, Japan
}

Received November 16, 2015; Accepted November 15, 2016

DOI: $10.3892 / \mathrm{mmr} .2016 .6066$

\begin{abstract}
The growth of biomedical research over the previous decades has been accompanied by an increase in the number, complexity and diversity of experimental animals developed as research tools, and inbred mice are some of the most widely used. However, thus far, no inbred mice have exhibited strong radioresistance for use in radiation-damage research. To develop a radioresistant mouse model, a female Japanese outbreeding strain ICR/JCL mouse was mated with a male Chinese inbred strain 615 mouse. From the F1 generation, the mouse line was maintained by brother-to-sister mating. A novel mouse strain was established over $>20$ continuous generations and designated the Institute of Radiation Medicine-2 (IRM-2) mouse. The biological characteristics, genetic characteristics and susceptibility to radiation of these mice were determined. The IRM-2 mice inherited traits from the parents, including strong reproductive capacity, stable physiological and biochemical indices and few differences among individuals. According to the genetic results, the IRM-2 mice exhibited homozygosity, isogenicity and consistency, in agreement with international standards for inbred strains. Radiosensitivity studies have previously suggested that the lethal dose $(\mathrm{LD})_{50}$ values for IRM-2 mice were 7.17 Gy (male) and 7.5 Gy (female), resulting in a dose reduction factor value of 1.39 (male) and 1.37 (female). The mortality of IRM-2 mice irradiated with 8 Gy total body irradiation was $15 \%$ at day 9 and $90 \%$ at day 15 after radiation. The number of nucleated cells in bone marrow, DNA content and colony-forming unit-spleen counts in IRM-2 mice after exposure to $\gamma$-ray irradiation were markedly higher than the
\end{abstract}

Correspondence to: Dr Qiang Liu, Tianjin Key Laboratory of Radiation Medicine and Molecular Nuclear Medicine, Institute of Radiation Medicine of Chinese Academy of Medical Science, 238 Baidi Road, Tianjin 300192, P.R. China

E-mail: liuqiang@irm-cams.ac.cn

Key words: inbred mouse strain, ionizing radiation, radiosensitivity, bone marrow, mouse model corresponding values for the parental strains, suggesting that the IRM-2 mice exhibit high resistance to ionizing radiation. Thus, it is suggested that this novel inbred mouse strain may be developed as an animal model of radioresistance for future use in radiation research.

\section{Introduction}

The rapid growth of biomedical research over the past several decades has seen a concomitant expansion in the number, complexity and diversity of experimental animals developed as research tools $(1,2)$ and inbred mice are among the most widely used. The accuracy and reproducibility of scientific studies can be greatly improved using inbred mice due to their particularly homozygous characteristics. At least 250 inbred mouse strains are commonly used worldwide, including $\mathrm{C} 57 \mathrm{BL} / 6 \mathrm{~J}, \mathrm{C} 3 \mathrm{H}, \mathrm{C} 3 \mathrm{HeB} / \mathrm{FeJ}, \mathrm{BALB} / \mathrm{c}, \mathrm{KK}, \mathrm{NC}, \mathrm{CBA} / \mathrm{J}$ and $\mathrm{CL} / \mathrm{Fr}$ mice (3-9). Different inbred mouse strains are required for various research types; $\mathrm{C} 57 \mathrm{BL} / 6 \mathrm{~J}$ is the most widespread substrain used for studying genetically engineered mice, $\mathrm{C} 3 \mathrm{H}$ is a common research model for the investigation of cancer, $\mathrm{C} 3 \mathrm{HeB} / \mathrm{FeJ}$ is a substrain of the $\mathrm{C} 3 \mathrm{H}$ strain with a high incidence of mammary tumors and $\mathrm{BALB} / \mathrm{c}$ mice are widely used for the production of monoclonal antibodies (4). To meet different research requirements, it is important to cultivate animal models with particular traits.

To best of our knowledge, no inbred mice have exhibited strong radioresistance for use in radiation-damage research at present. In the current study a variety of mice strains, including LACA, NIH (data not shown), ICR/JCL and Kunming (data not shown) mice, were tested in the attempt to develop a radioresistant animal model. The desired characteristics were challenging to achieve due to the biological characteristics of these mice. For example, inbred mice had stable physiological and biochemical indicators however exhibited low radioresistance; thus, the animals did not survive exposure to the required radiation dose. Hybrid mice exhibited high radioresistance (10), however their physiological and biochemical indicators were unstable, resulting in poor reproducibility. It was noted that the radioresistance of the Japan outbreeding mouse strain ICR/JCL to ionizing radiation was higher than 
that of other hybrid mice and that the Chinese inbred mouse 615 had stable hematopoietic indicators and was sensitive to radiation. According to the laws of genetics, offspring can combine the biological advantages of their parents. The aim of the present study was to generate a new inbred mouse strain with high radioresistance and stable genetic characteristics.

To develop an inbred mouse with the desired characteristics, a female ICR/JCL mouse (white fur) was crossed with a male 615 mouse (black fur). A new mouse strain (with cinnamon-colored fur) was established through more than 20 continuous generations and was termed Institute of Radiation Medicine-2 (IRM-2) mouse. The biological characteristics of the IRM-2 mouse, including reproductive capacity, physiological and biochemical indices and genetic characteristics, were determined. In addition, the radiosensitivity of the IRM-2 mouse to $\gamma$-ray was examined using a lethal dose (LD) ${ }_{50}$ test and assays of the hematopoietic function of bone marrow.

\section{Materials and methods}

Development of IRM-2 mice. Japanese outbreeding-strain female ICR/JCL mice and Chinese inbred-strain male 615 mice (weight: $20 \pm 2 \mathrm{~g}$ ) were purchased from the Academy of Military Medical Sciences (Beijing, China). They were maintained under controlled laboratory conditions at a temperature of $23 \pm 2^{\circ} \mathrm{C}$ and humidity of $55 \pm 5 \%$ with a controlled light cycle (14 h of light and $10 \mathrm{~h}$ of darkness). A female ICR/JCL mouse was crossed with a male 615 mouse to produce F1 hybrids. Subsequently, the F1 mice were further interbred by brother-sister mating to obtain an F2 generation. A novel mouse strain was established through $>20$ continuous generations and termed the IRM-2 mouse. The mice were bred at an animal care facility certified by Tianjin Management Committee of Laboratory Animals in the Institute of Radiation Medicine at Peking Union Medical College (Beijing, China). The experimental protocol was approved by the China Institutional Ethics Review Committee for Animal Experimentation. IRM-2 mice, 615 mice and ICR/JCL mice used were 8-10 weeks old.

Ionizing radiation. Mice were exposed to ionizing radiation (IR) in a Gammacell-40 ${ }^{137}$ Cesium $\gamma$ irradiator (Atomic Energy of Canada Inc., Chalk River, ON, Canada) at a rate of $0.882 \mathrm{~Gy} / \mathrm{min}$. After irradiation, the mice were returned to the certified animal facility.

Organ-coefficient measurement. Mice were anaesthetized by intraperitoneal administration of $300 \mathrm{mg} / \mathrm{kg}$ chloral hydrate solution (Baomanbio, Inc., Shanghai, China). After sacrifice by cervical dislocation, the hearts, livers, spleens, lungs, kidneys and thymuses of the mice were dissected out and weighed. Each organ coefficient was calculated as organ coefficient=organ weight/body weight $\times 100 \%$.

Peripheral blood cells and bone marrow cell (BMC) counts. Whole blood was drawn from the orbital sinuses of mice and used within $30 \mathrm{~min}$ of collection to perform white blood cell (WBC), red blood cell (RBC) and platelet (PLT) counts for each sample, using a hemocytometer (Sysmex pocH-100i; Sysmex Corporation, Kobe, Japan). After the mice were euthanized by cervical dislocation, BMCs were flushed from the mouse femurs as described previously (11), counted using the hemocytometer (Sysmex pocH-100i).

Measurement of biochemical indices. Following a $12 \mathrm{~h}$ fast, the mice were euthanized by cervical dislocation. Whole blood was sampled by eyeball extirpation and centrifuged for $10 \mathrm{~min}$ at 2,500 $\mathrm{x} g$ at room temperature. The concentrations of serum biochemical indices were measured using a semi-automatic biochemical analyzer (VITALAB-II; Vital Scientific N.V., Dieren, The Netherlands); the indices used included blood glucose, total cholesterol, triglycerides, aspartate aminotransferase, alanine aminotransferase, alkaline phosphatase, total protein, albumin, blood urea nitrogen, creatinine, calcium, phosphorus and total bilirubin.

\section{Gene homogeneity characterization}

Coat-color gene test. An IRM-2 mouse was mated with an albino BALB/c mouse (genotype AAbbccDD) with known genes. The coat-color genes of the IRM-2 mouse were judged based on the coat color of F1-generation mice.

Biochemical markers test. IRM-2 mice were randomly selected from the F23 and F38 generations of the population. Biochemical markers were detected according to the National Standard of China GB/T14927.1-2001 on Laboratory Animal Genetic Monitoring: Methods for Biochemical Markers of Inbred Mice and Rats (12).

Chromosomal aberrations analysis and chromosome $G$-banding karyotype. Mice were administered an intraperitoneal dose of $7 \mu \mathrm{g} / \mathrm{g}$ body weight colchicine (Sigma-Aldrich; Merck Millipore, Darmstadt, Germany) and euthanized after $4 \mathrm{~h}$. Bone-marrow cells were flushed from the femurs using Hank's balanced salt solution. The cells were treated with a pre-warmed hypotonic lysis solution $(0.075 \mathrm{M} \mathrm{KCl})$ at $37^{\circ} \mathrm{C}$ for $30 \mathrm{~min}$. The cells were then fixed with Carnoy's solution [3:1 (v/v) methanol/glacial acetic acid] for $20 \mathrm{~min}$ at room temperature. Metaphase slides were prepared by dropping the cells onto glass slides, which were placed into an oven at $60^{\circ} \mathrm{C}$ for $4 \mathrm{~h}$, digested with $0.03 \%$ trypsin-EDTA solution for 20-30 sec and then stained with Giemsa. Spontaneous chromosomal aberrations based on 1,200 metaphases were examined under the microscope (Eclipse 50 i, Nikon Corporation, Tokyo, Japan). Chromosomes were identified based on unique G-banding patterns and the chromosome karyotypes were drawn (original magnification, $\mathrm{x} 400$ ).

Relative chromosome length. A total of five mitotic metaphases of well-dispersed and moderate length chromosomes of mice were selected under the microscope and were imaged. Chromosomes of five metaphases were developed and enlarged. The length of the straighter chromosome of each pair of homologous chromosomes was measured with a vernier caliper and the total length of all chromosomes was calculated. The relative chromosome length of each chromosome karyotype was calculated as relative chromosome length=(length of each chromosome/total length of all chromosomes)x $100 \%$. The relative chromosome length of the five chromosome karyotype was averaged. 
Table I. Organ coefficients of mice $(\%)$ presented as the mean \pm standard deviation; $\mathrm{n}=40$.

\begin{tabular}{|c|c|c|c|c|c|c|}
\hline \multirow[b]{2}{*}{ Organ } & \multicolumn{2}{|c|}{ IRM-2 } & \multicolumn{2}{|c|}{615} & \multicolumn{2}{|c|}{ ICR/JCL } \\
\hline & Male & Female & Male & Female & Male & Female \\
\hline Thymus & $0.13 \pm 0.03$ & $0.28 \pm 0.05$ & $0.21 \pm 0.02^{\mathrm{a}}$ & $0.52 \pm 0.10^{\mathrm{a}}$ & $0.25 \pm 0.1^{\mathrm{a}}$ & $0.45 \pm 0.12^{\mathrm{a}}$ \\
\hline Spleen & $0.33 \pm 0.04$ & $0.43 \pm 0.04$ & $0.42 \pm 0.10$ & $0.41 \pm 0.11$ & $0.42 \pm 0.09$ & $0.45 \pm 0.13$ \\
\hline Heart & $0.47 \pm 0.03$ & $0.47 \pm 0.02$ & $0.53 \pm 0.11$ & $0.55 \pm 0.12$ & $0.58 \pm 0.13$ & $0.55 \pm 0.15$ \\
\hline Lung & $0.57 \pm 0.06$ & $0.68 \pm 0.05$ & $0.72 \pm 0.12$ & $0.85 \pm 0.13$ & $0.76 \pm 0.15$ & $0.85 \pm 0.18$ \\
\hline Kidney & $1.64 \pm 0.04$ & $1.23 \pm 0.05$ & $1.23 \pm 0.13$ & $1.03 \pm 0.12$ & $1.50 \pm 0.15$ & $1.28 \pm 0.12$ \\
\hline Liver & $5.53 \pm 0.35$ & $4.68 \pm 0.31$ & $4.22 \pm 0.50$ & $4.25 \pm 0.53$ & $6.01 \pm 0.58$ & $5.20 \pm 0.50$ \\
\hline
\end{tabular}

${ }^{\mathrm{a}} \mathrm{P}<0.01$ vs. IRM- 2 mice of the same gender.

A
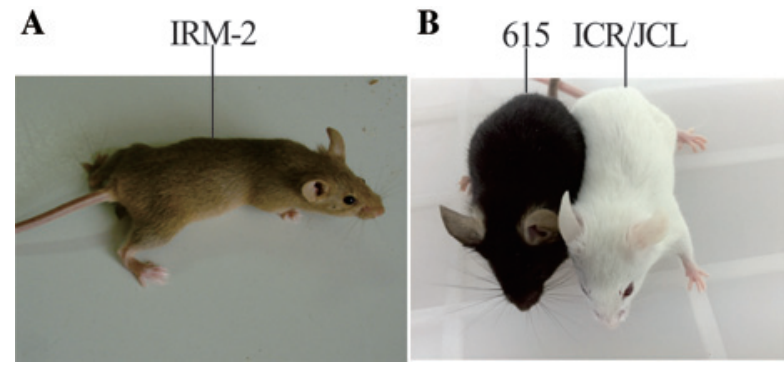

Figure 1. An IRM-2 mouse and its parents. A novel mouse strain was derived from crosses between ICR/JCL mouse (maternal line) and 615 mouse (parental line) and designated the IRM-2 mouse through more than 20 continuous breeding generations. (A) IRM-2 mouse and (B) ICR/JCL mouse (maternal strain) and 615 mouse (parental strain).

Lethal dose, $50 \%$ test and dose reduction factor analysis. Mice were exposed to a total body irradiation of 0-10 Gy (absolute lethal dose) ${ }^{137} \mathrm{Cs} \gamma$-rays. The mortality of each mouse dose group was recorded 30 days after irradiation and the $\mathrm{LD}_{50}$ was calculated. The radioresistant characteristics of mice were expressed as the dose reduction factor (DRF). The DRF was calculated as the ratio of radiation dose required to produce the same biological effect of median lethal dose of 30 days $\left(\mathrm{LD}_{50} / 30\right)$. Mice were observed for 30 days after having been exposed to an $\mathrm{LD}_{50}$ dose of radiation to determine $\mathrm{LD}_{50} / 30$. Surviving mice were sacrificed at day 31 .

Survival curve. Mice were exposed to a total body irradiation of $8 \mathrm{~Gy}{ }^{137} \mathrm{Cs} \gamma$-rays. The number of surviving mice was recorded continuously for 15 days after irradiation and the survival curve of the mice was determined.

DNA content of BMCs. BMCs were flushed from mouse femurs using $0.005 \mathrm{~mol} / 1 \mathrm{CaCl}_{2}$ after the mice were euthanized by cervical dislocation. The cells were treated with a $0.2 \mathrm{~mol} / 1 \mathrm{HClO}_{4}$ solution at $90^{\circ} \mathrm{C}$ for $15 \mathrm{~min}$ and filtered. The optical density of the BMCs was measured at $286 \mathrm{~nm}$ using a UV spectrophotometer (Thermo Fisher Scientific, Inc., Waltham, MA, USA).

Colony-forming unit-spleen (CFU-S) counting. Spleens were harvested from the abdominal cavity of mice after euthanization by cervical dislocation and stained using picric acid. The number of CFU-S was determined by macrography using a simple magnifier. One nodule on the spleen surface that was apparent to the naked eye was considered as one CFU-S. One CFU-S was treated as one CFU-S unit.

Statistical analysis. All experiments were performed at least twice. Data are presented as mean \pm standard deviation. Differences between groups were calculated using Student's $t$-test. $\mathrm{P}<0.05$ was considered to indicate a statistically significant difference.

\section{Results}

The growth and development of IRM-2 mice. The IRM-2 mice were derived from crosses between ICR/JCL and 615 parents (Fig. 1). The specific characteristics of the mice included cinnamon-colored fur, large semicircular ears, protruding eyes and black irises. The breeding performance of IRM-2 mice was characterized as follows: i) Number of pups per female/lifetime $=45$; ii) litter size $=7.8$; iii) number of litters $=5.8$; iv) age at first productive mating $=7.5$ weeks; v) age at last productive mating $=32.6$ weeks; and vi) life span=77.1 weeks.

The body weight, body length and tail length of the IRM-2 mice were measured from birth to 70 days. During the period of rapid growth from 25 to 40 days old, the body weight of the mice increased twice as much as that between birth and 25 days (Fig. 2A). After this period, the increase in body weight slowed and the mice reached their maximum weight of $25 \mathrm{~g} 70$ days following birth. The increases in body length and tail length were similar to that of body weight (Fig. 2B and C). The growth curves were plotted for male and female mice separately, however, no significant differences were identified between the males and females.

Organ coefficients of mice. The organ coefficients of IRM-2, 615 and ICR/JCL mice were measured. The organ coefficients of the thymus of same-gender IRM-2 mice was significantly smaller than that of same-gender 615 mice and ICR/JCL mice $(\mathrm{P}<0.0001$; Table I). No significant differences were identified in the organ coefficients of other organs, with the exception of the thymus, between the males and females of IRM-2 mice, 615 mice and ICR/JCL mice (Table I). 
Table II. Biochemical indices of mice presented as the mean \pm standard deviation; $\mathrm{n}=10$.

\begin{tabular}{|c|c|c|c|c|c|c|c|}
\hline \multirow[b]{2}{*}{ Index } & \multirow[b]{2}{*}{ Unit } & \multicolumn{2}{|c|}{ IRM-2 } & \multicolumn{2}{|c|}{615} & \multicolumn{2}{|c|}{ ICR/JCL } \\
\hline & & Male & Female & Male & Female & Male & Female \\
\hline ALB & $\mathrm{g} / \mathrm{l}$ & $18.95 \pm 1.06$ & $21.22 \pm 1.11$ & $20.26 \pm 1.89$ & $23.52 \pm 2.06$ & $21.65 \pm 1.69$ & $24.21 \pm 1.35$ \\
\hline ALP & $\mathrm{U} / 1$ & $116.50 \pm 10.52$ & $117.70 \pm 13.06$ & $115.52 \pm 12.27$ & $120.72 \pm 12.19$ & $118.16 \pm 11.62$ & $121.05 \pm 12.80$ \\
\hline ALT & $\mathrm{U} / 1$ & $44.10 \pm 6.21$ & $39.40 \pm 4.88$ & $40.23 \pm 5.08$ & $42.76 \pm 4.65$ & $45.12 \pm 6.72$ & $44.63 \pm 5.06$ \\
\hline AST & $\mathrm{U} / 1$ & $113.60 \pm 11.16$ & $128.20 \pm 12.38$ & $116.63 \pm 12.45$ & $130.23 \pm 13.78$ & $118.25 \pm 12.86$ & $131.50 \pm 14.03$ \\
\hline BUN & $\mathrm{mmol} / \mathrm{l}$ & $5.66 \pm 0.38$ & $5.28 \pm 1.19$ & $6.03 \pm 0.56$ & $6.12 \pm 0.95$ & $5.82 \pm 0.67$ & $6.05 \pm 1.15$ \\
\hline $\mathrm{Ca}$ & $\mathrm{mg} / \mathrm{dl}$ & $7.02 \pm 0.61$ & $6.80 \pm 1.03$ & $6.91 \pm 0.86$ & $7.26 \pm 1.06$ & $7.15 \pm 0.95$ & $6.96 \pm 1.12$ \\
\hline $\mathrm{CHO}$ & $\mathrm{mmol} / \mathrm{l}$ & $3.59 \pm 0.30$ & $3.94 \pm 0.25$ & $3.78 \pm 0.58$ & $4.02 \pm 0.65$ & $3.96 \pm 0.67$ & $4.10 \pm 0.87$ \\
\hline Cre & $\mu \mathrm{mol} / 1$ & $51.08 \pm 6.02$ & $52.19 \pm 7.26$ & $52.39 \pm 8.22$ & $54.38 \pm 8.26$ & $52.81 \pm 7.56$ & $55.63 \pm 7.95$ \\
\hline GLU & $\mathrm{mmol} / \mathrm{l}$ & $4.77 \pm 0.80$ & $4.19 \pm 0.81$ & $4.23 \pm 1.02$ & $4.65 \pm 0.95$ & $5.07 \pm 0.86$ & $4.89 \pm 1.23$ \\
\hline $\mathrm{P}$ & $\mathrm{mg} / \mathrm{dl}$ & $7.77 \pm 0.87$ & $7.40 \pm 0.66$ & $7.50 \pm 0.93$ & $8.26 \pm 1.05$ & $7.56 \pm 0.86$ & $7.90 \pm 0.98$ \\
\hline TBIL & $\mu \mathrm{mol} / 1$ & $3.83 \pm 0.96$ & $3.87 \pm 1.22$ & $3.72 \pm 0.93$ & $4.15 \pm 0.88$ & $3.90 \pm 1.02$ & $4.21 \pm 1.16$ \\
\hline TG & $\mathrm{mmol} / \mathrm{l}$ & $0.78 \pm 0.13$ & $0.79 \pm 0.15$ & $0.85 \pm 0.23$ & $0.76 \pm 0.25$ & $0.87 \pm 0.32$ & $0.90 \pm 0.35$ \\
\hline $\mathrm{TP}$ & $\mathrm{g} / 1$ & $28.18 \pm 1.25$ & $29.45 \pm 3.95$ & $26.65 \pm 2.72$ & $30.05 \pm 4.17$ & $28.85 \pm 2.53$ & $32.58 \pm 3.63$ \\
\hline
\end{tabular}

ALB, albumin; ALP, alkaline phosphatase; ALT, alanine aminotransferase; AST, aspartate aminotransferase; BUN, blood urea nitrogen; Ca, calcium; CHO, total cholesterol; Cre, creatinine; GLU, blood glucose; P, phosphorus; TBIL, total bilirubin; TG, triglycerides; TP, total protein .
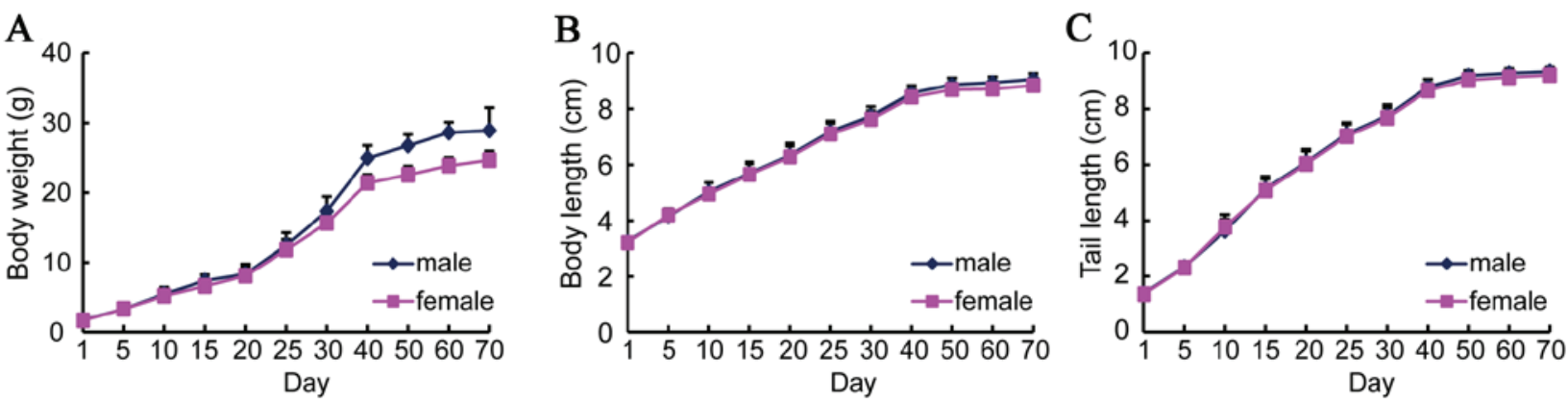

Figure 2. Growth curves of IRM-2 mice. The growth and development of IRM-2 mice was determined from birth and the growth curves were plotted (n=40). The increases in (A) body weight, (B) body length and (C) tail length were measured from birth to 70 days.

Hematic and bone-marrow phases of IRM-2 mice. To investigate the biological characteristics of IRM-2 mice, images of peripheral blood and bone marrow were recorded. No significant differences were identified in peripheral WBC, RBC and PLT or in the number of BMCs between 7 and 14-week-old IRM-2 mice (Fig. 3).

Serum biochemical indices of mice. Biochemical indices are important biological indicators for characterizing laboratory animals. A total of 13 serum biochemical indices of male and female IRM-2, 615 and ICR/JCL mice were determined. All the tested indices were stable; the individual differences of those indices were low and no differences were identified between male and female IRM-2 mice, 615 mice or ICR/JCL mice (Table II).

Coat-color gene test of IRM-2 mice. To characterize the coat genotype of IRM-2 mice, an IRM-2 mouse was mated with an albino BALB/c mouse (genotype AAbbccDD) with known genes. The coat-color genes of the IRM-2 mouse were judged based on the coat color of F1-generation mice. The coat color of all offspring (six litters) was cinnamon, indistinguishable from that of IRM-2 mice (Fig. 4). The results demonstrated that the coat-color gene of IRM-2 mice was homozygous and the coat genotype was AAbbCCDD.

Biochemical markers of mice. The biochemical markers of IRM-2 mice were measured at generations F23 and F38 to confirm the genetic quality of the mice. No polymorphisms or mutant genes were observed in any of the biochemical markers tested. A total of 20 markers were detected for the IRM-2 mice at generation F23. Of the 20 markers analyzed, seven differed between the IRM-2 mice and the 615 mice, including alkaline phosphatase 1 (Akp1), carbonic anhydrase 2 (Car2), kidney catalase 2 (Ce2), major urinary protein 1 (Mup1), peptidase 3 (Pep3), phosphoglucomutase 1 (Pgm1) and tosyl arginine methylesterase 1 (Tam1) (Table III). Of the 20 markers analyzed, 12 were measured in the IRM- 2 mice at generation F38. Of these 12, four differed among the IRM-2, 615 and ICR/JCL mice, including Akp1, Car2, Ce2 and Pgm1 

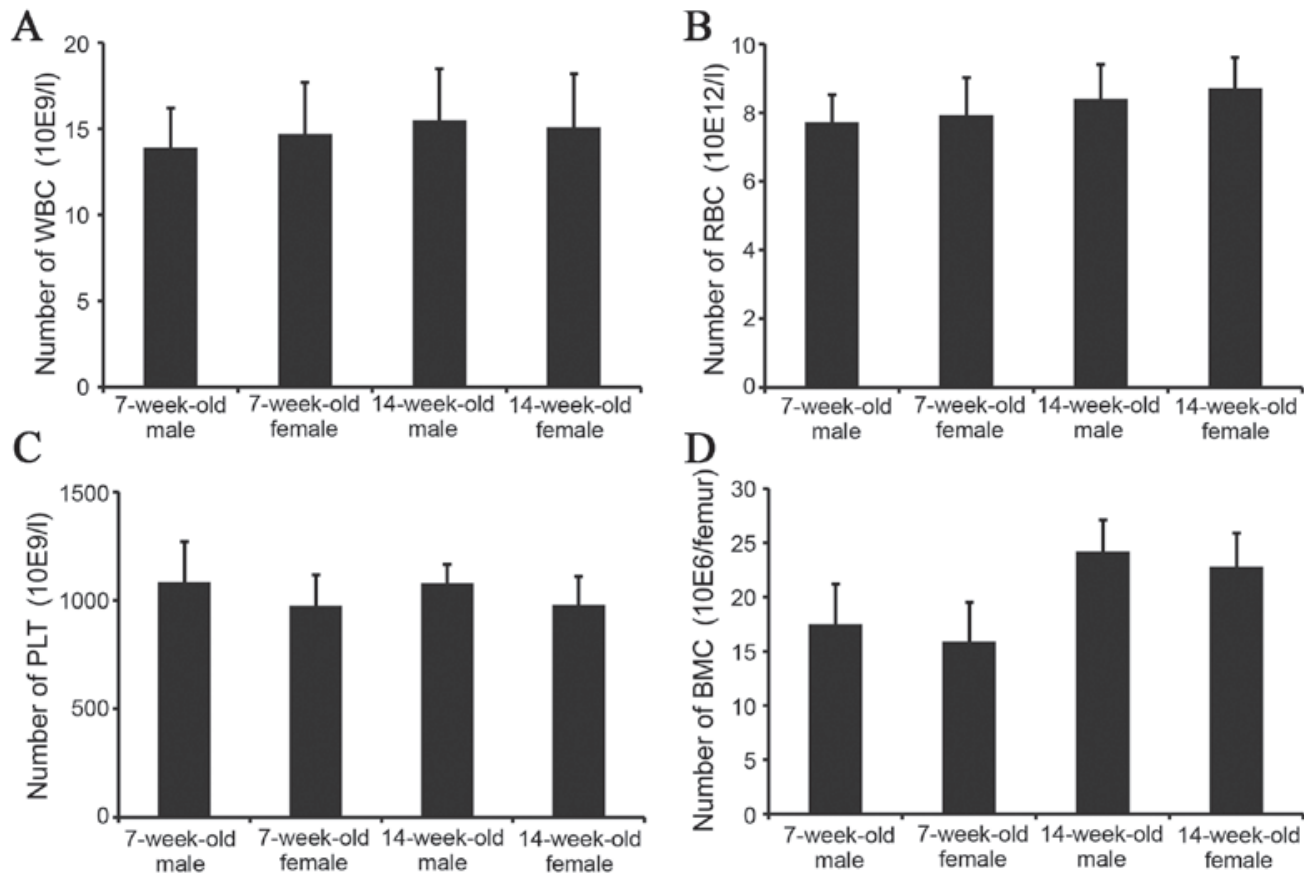

Figure 3. Peripheral blood and bone-marrow characteristics of IRM-2 mice. Whole blood was drawn from the orbital sinuses of 7-week-old and 14-week-old male and female IRM-2 mice to count peripheral blood cells. BMCs were flushed from 7-week-old and 14-week-old male and female IRM-2 mice femurs and counted ( $\mathrm{n}=10$ ). (A) Peripheral WBC count of IRM-2 mice. (B) Peripheral RBC count of IRM-2 mice. (C) Peripheral PLT count of IRM-2 mice. (D) BMC count of IRM-2 mice. BMCs, bone-marrow cells; WBC, white blood cell; RBC, red blood cell; PLT, platelet.

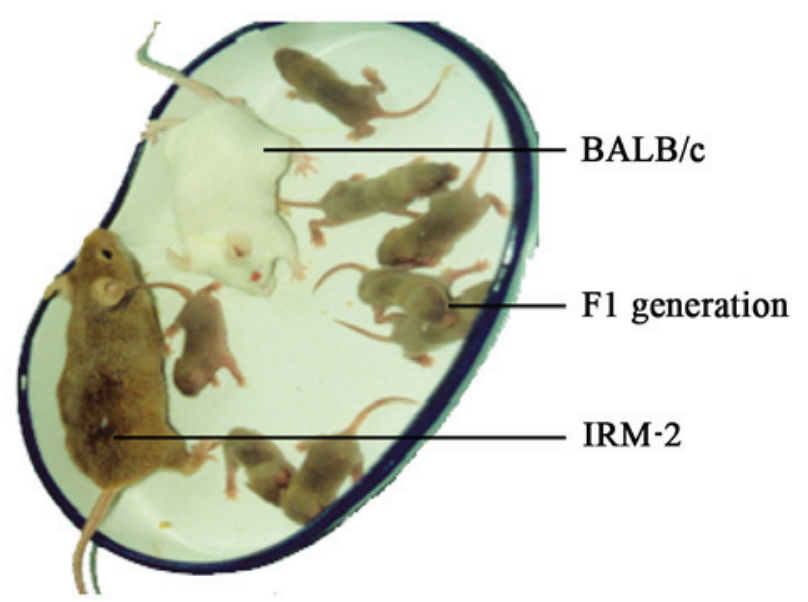

Figure 4. Coat color of F1-generation mice hybridized from IRM-2 and BALB/c parents. An IRM-2 mouse was mated with a BALB/c mouse with known genes. Coat genotype of IRM-2 mice was identified based on the coat color of two-week-old F1-generation mice.

(these were among the seven markers that differed in the F23 generation; Table IV). These results indicate that the IRM-2 mice had their own specific genotype and were homozygous.

Chromosome analysis of IRM-2 mice. The cytogenetic analysis of chromosomes is an essential part of experimental animal characterization. The chromosomes of the IRM-2 mice were identified based on differences in the shading of the G-bands (Fig. 5A). The IRM-2 mice had 40 chromosomes, all of which were telocentric. The G-banding technique was applied to karyotype the IRM-2 mice as follows: Male 40, XY (Fig. 5B); female 40, XX.
The length of homologous chromosomes in the IRM-2 mice was measured and the relative length of the chromosomes was calculated. The relative length of all the chromosomes differed: Chromosome 1 was the longest and chromosomes 19 and $\mathrm{Y}$ were the shortest (Fig. 5C).

In addition, spontaneous chromosomal aberrations based on 1,200 metaphases were examined. The chromosomal aberration frequency of the IRM-2 mice was the lowest, $0.5 \%$, compared with that of 615 mice and ICR/JCL mice, 1.2 and $1.3 \%$ respectively (Table $\mathrm{V}$ ).

$L D_{50}$ of mice strains following exposure to $\gamma$-ray irradiation and DRF analysis. $\mathrm{LD}_{50}$ of the ICR/JCL mice and 615 mice were calculated for $5.44 \mathrm{~Gy}$ (male), $5.93 \mathrm{~Gy}$ (female) and $5.73 \mathrm{~Gy}$ (male), $6.02 \mathrm{~Gy}$ (female) respectively (Fig. 6). However, it was increased to $7.17 \mathrm{~Gy}$ (male) and 7.5 Gy (female) for the IRM-2 mice, significantly higher than those of the parents (the 615 mice and ICR/JCL mice), BALB/c mice or C57BL/6 mice (P<0.0001; Fig. 6).

The DRF calculated by $\mathrm{LD}_{50} / 30$ for the ICR/JCL mice and 615 mice compared with the C57BL/6 mice had a value of 1.05 (male), 1.08 (female) and 1.11 (male), 1.10 (female) respectively; the IRM-2 mice had the maximum DRF of 1.39 (male) and 1.37 (female).

Survival curve. To test the radioresistance of the IRM-2 mice, the survival curve of mice after total body irradiation with $8 \mathrm{~Gy}$ doses of ${ }^{137} \mathrm{Cs} \gamma$-rays was plotted. As demonstrated in Fig. 7, total body radiation resulted in 55 and $45 \%$ mortality in 615 mice and ICR/JCL mice respectively by day 9 after irradiation and the rest of the mice died at day 15 after irradiation. The mortality of IRM-2 mice was $15 \%$ at day 9 and $90 \%$ at day 15 . The survival rate of IRM-2 mice was higher 
Table III. Biochemical markers of IRM-2 mice at generation $\mathrm{F} 23(\mathrm{n}=8)$.

\begin{tabular}{lccc}
\hline & \multicolumn{3}{c}{ Genotype } \\
\cline { 2 - 4 } Biochemical markers & IRM-2 & 615 & ICR/JCL \\
\hline Akp1 & $\mathrm{b}$ & $\mathrm{a}$ & $\mathrm{a}$ \\
Amy1 & $\mathrm{a}$ & $\mathrm{a}$ & $\mathrm{a}$ \\
Car2 & $\mathrm{b}$ & $\mathrm{a}$ & $\mathrm{b}$ \\
Ce2 & $\mathrm{a}$ & $\mathrm{b}$ & $\mathrm{b}$ \\
Es1 & $\mathrm{b}$ & $\mathrm{b}$ & $\mathrm{b}$ \\
Es2 & $\mathrm{b}$ & $\mathrm{b}$ & $\mathrm{b}$ \\
Es3 & $\mathrm{c}$ & $\mathrm{c}$ & $\mathrm{c}$ \\
Es10 & $\mathrm{a}$ & $\mathrm{a}$ & $\mathrm{a}$ \\
Es11 & $\mathrm{a}$ & $\mathrm{a}$ & $\mathrm{a}$ \\
Gpd1 & $\mathrm{b}$ & $\mathrm{b}$ & $\mathrm{b}$ \\
Gpi1 & $\mathrm{a}$ & $\mathrm{a}$ & $\mathrm{a}$ \\
Hbb & $\mathrm{s}$ & $\mathrm{s}$ & $\mathrm{s}$ \\
Idh1 & $\mathrm{a}$ & $\mathrm{a}$ & $\mathrm{a}$ \\
Ldr1 & $\mathrm{a}$ & $\mathrm{a}$ & $\mathrm{a}$ \\
Mup1 & $\mathrm{a}$ & $\mathrm{b}$ & $\mathrm{a}$ \\
Pep3 & $\mathrm{b}$ & $\mathrm{a}$ & $\mathrm{a}$ \\
Pgm1 & $\mathrm{a}$ & $\mathrm{b}$ & $\mathrm{b}$ \\
Pgm2 & $\mathrm{a}$ & $\mathrm{a}$ & $\mathrm{a}$ \\
Tam1 & $\mathrm{a}$ & $\mathrm{c}$ & $\mathrm{c}$ \\
Trf & $\mathrm{b}$ & $\mathrm{b}$ & $\mathrm{b}$ \\
\hline
\end{tabular}

Akp1, alkaline phosphatase 1; Amy1, amylase 1; Car2, carbonic anhydrase 2; Ce2, kidney catalase 2; Es1, esterase 1; Es2, esterase 2; Es3, esterase3; Es10, esterase 10; Es11, esterase 11; Gpd1, glycerol-3-phosphate dehydrogenase 1; Gpi1, glucose phosphate isomerase 1; Hbb, hemoglobin beta chain complex; Idh1, isocitrate dehydrogenase 1; Ldr1, lactate dehydrogenase regulator 1; Mup1, major urinary protein 1; Pep3, peptidase 3; Pgm1, phosphoglucomutase 1; Pgm2, phosphoglucomutase 2; Tam1, tosyl arginine methylesterase 1; Trf, transferrin; a, fast-migrating anodic band or slow-migrating cathodic band or slow-migrating anodic band; b, slow-migrating anodic band or fast-migrating cathodic band or fast-migrating anodic band; c, slowest-migrating anodic band; s, fast-migrating anodic band.

than that of ICR/JCL mice and 615 mice 9 to 15 days after irradiation $(\mathrm{P}=0.0405$ vs. ICR $/ \mathrm{JCL}$ mice and $\mathrm{P}=0.0112$ vs. 615 mice; Fig. 7).

Alterations in leukocytes and the hemopoietic system following exposure to radiation. Leukocyte counts decreased rapidly in the three mice strains 2 days after exposure to $4 \mathrm{~Gy}$ radiation, falling to $\sim 20 \%$ of their pre-irradiation value; subsequently, the counts gradually increased and were restored to $\sim 45 \%$ of their pre-irradiation value at 21 days (Fig. 8A). The number of leukocytes in the IRM-2 mice was higher than that in the ICR/JCL mice and 615 mice 12-21 days after the radiation exposure $(\mathrm{P}=0.0396$ vs. ICR $/ \mathrm{JCL}$ mice and $\mathrm{P}=0.0076$ vs. 615 mice; Fig. 8A).

To detect the hematopoietic function of mice after $4 \mathrm{~Gy}$ radiation, changes in the numbers of the bone marrow
Table IV. Biochemical markers of IRM-2 mice at generation F38 ( $n=8)$.

\begin{tabular}{lccc}
\hline & \multicolumn{3}{c}{ Genotype } \\
\cline { 2 - 4 } Biochemical markers & IRM-2 & 615 & ICR/JCL \\
\hline Akp1 & $\mathrm{b}$ & $\mathrm{a}$ & $\mathrm{a}$ \\
Car2 & $\mathrm{b}$ & $\mathrm{a}$ & $\mathrm{b}$ \\
Ce2 & $\mathrm{a}$ & $\mathrm{b}$ & $\mathrm{b}$ \\
Es1 & $\mathrm{b}$ & $\mathrm{b}$ & $\mathrm{b}$ \\
Es3 & $\mathrm{c}$ & $\mathrm{c}$ & $\mathrm{c}$ \\
Es11 & $\mathrm{a}$ & $\mathrm{a}$ & $\mathrm{a}$ \\
Gpd1 & $\mathrm{b}$ & $\mathrm{b}$ & $\mathrm{b}$ \\
Gpi1 & $\mathrm{a}$ & $\mathrm{a}$ & $\mathrm{a}$ \\
Hbb & $\mathrm{s}$ & $\mathrm{s}$ & $\mathrm{s}$ \\
Idh1 & $\mathrm{a}$ & $\mathrm{a}$ & $\mathrm{a}$ \\
Pgm1 & $\mathrm{a}$ & $\mathrm{b}$ & $\mathrm{b}$ \\
Trf & $\mathrm{b}$ & $\mathrm{b}$ & $\mathrm{b}$
\end{tabular}

Akp1, alkaline phosphatase 1; Amy 1, amylase 1; Car2, carbonic anhydrase 2; Ce2, kidney catalase 2; Es1, esterase 1; Es3, esterase3; Es11, esterase 11; Gpd1, glycerol-3-phosphate dehydrogenase 1; Gpi1, glucose phosphate isomerase 1; Hbb, hemoglobin beta chain complex; Idh1, isocitrate dehydrogenase 1; Pgm1, phosphoglucomutase 1; Trf, transferrin; a, fast-migrating anodic band or slow-migrating cathodic band or slow-migrating anodic band; b, slow-migrating anodic band or fast-migrating cathodic band or fast-migrating anodic band; $c$, slowest-migrating anodic band; s, fast-migrating anodic band.

nucleated cells (BMNCs), and the DNA content of BMCs and CFU-S were determined. The number of BMNCs in the three mice strains was decreased significantly to $\sim 20 \%$ of the pre-irradiation value 2 days after radiation, after which the value began to increase again in the following days. A rapid increase in the BMNC count was identified 2-9 days after irradiation, reaching $\sim 80 \%$ of the pre-irradiation value and remaining at a stable level (Fig. $8 \mathrm{~B}$ ). The number of BMNC of the IRM-2 mice was significantly higher than that of the ICR/JCL and 615 mice 9-21 days after irradiation $(\mathrm{P}<0.0001$ vs. ICR/JCL and 615 mice; Fig. $8 \mathrm{~B}$ ), indicating that the hematopoietic function of the bone marrow of IRM-2 mice recovered quickly.

The DNA content of the BMCs of the three mice strains decreased significantly 2 days after 4 Gy radiation, decreasing to $\sim 20 \%$ of the pre-irradiation value. The DNA content then recovered rapidly, reaching $\sim 90 \%$ of the pre-irradiation value on day 9 and decreasing slightly in the following days (Fig. 8C). The DNA content of the IRM-2 mice was significantly higher than that of the ICR/JCL and 615 mice 9-21 days after irradiation $(\mathrm{P}=0.0213$ vs. ICR/JCL mice and $\mathrm{P}=0.0447$ vs. 615 mice; Fig. 8C), similarly suggesting that the hematopoietic function of the bone marrow of the IRM-2 mice recovered quickly.

CFU-S were not identified in the three mice strains prior to exposure to radiation and developed 5 days after 4 Gy irradiation. CFU-S counts reached maximum values 9 days after irradiation and decreased rapidly over the following days. Few CFU-S were observed 21 days after radiation (Fig. 8D). The 
Table V. Spontaneous chromosomal aberration frequency of mice $(n=20)$.

\begin{tabular}{lcccccc}
\hline & & \multicolumn{3}{c}{ Chromosomal aberration } & & \\
\cline { 3 - 6 } Mice & $\begin{array}{c}\text { No. of } \\
\text { metaphases }\end{array}$ & $\begin{array}{c}\text { Chromosomal } \\
\text { fracture }\end{array}$ & Acentric & $\begin{array}{c}\text { Unbalanced } \\
\text { translocation }\end{array}$ & $\begin{array}{c}\text { Total } \\
\text { aberration }\end{array}$ & $\begin{array}{c}\text { Aberration } \\
\text { frequency (\%) }\end{array}$ \\
\hline IRM-2 & 1,200 & 4 & 1 & 1 & 6 & 0.5 \\
615 & 1,200 & 10 & 2 & 2 & 14 & 1.2 \\
ICR/JCL & 1,200 & 12 & 3 & 1 & 16 & 1.3 \\
\hline
\end{tabular}

A

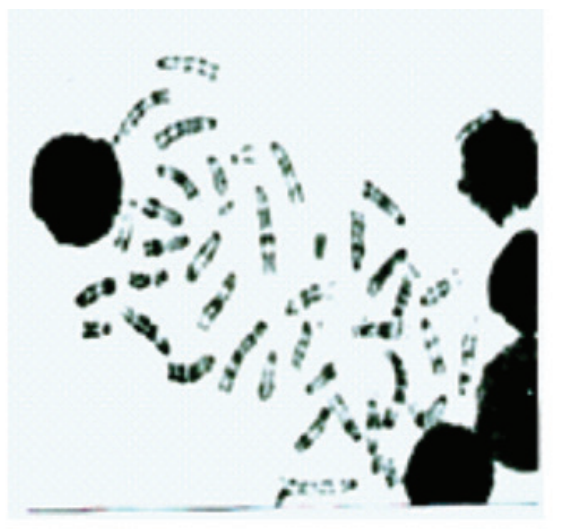

$\mathrm{C}_{8}$

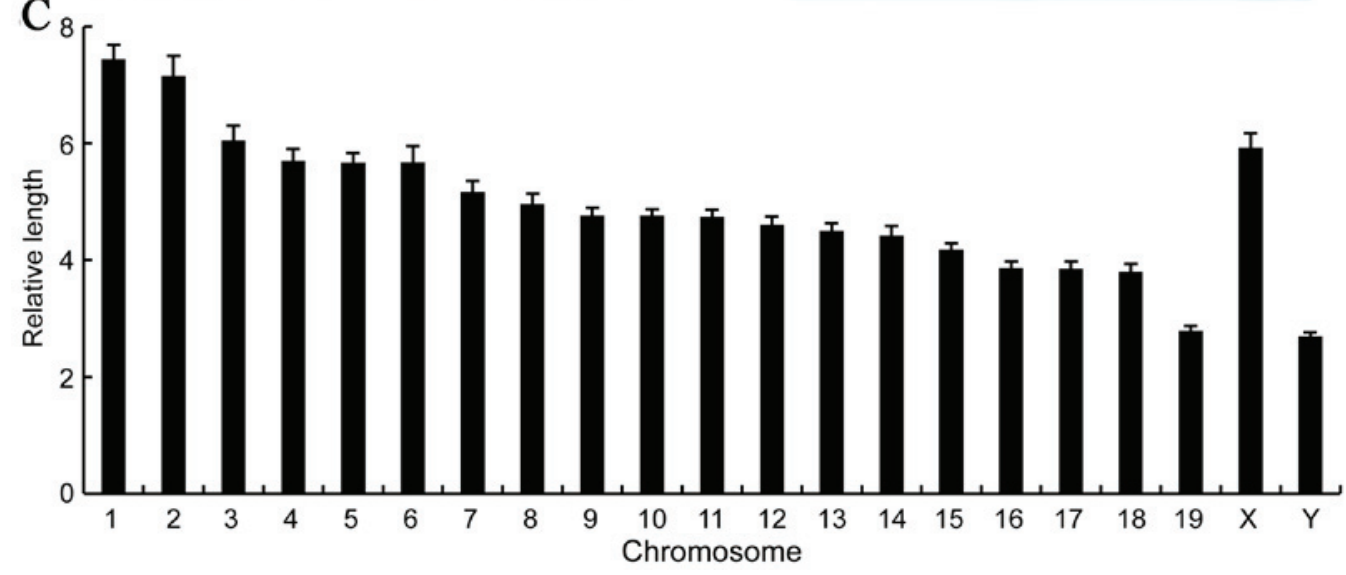

\section{B}

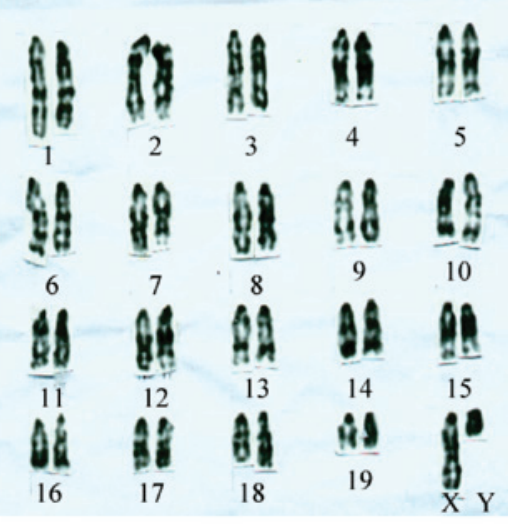

Figure 5. Chromosome karyotype and relative chromosome length in the IRM-2 mice. IRM-2 mice were administered an intraperitoneal dose of $7 \mu \mathrm{g} / \mathrm{g}$ body weight colchicine and euthanized after $4 \mathrm{~h}$. (A) Bone-marrow cells were flushed from the femurs. Metaphase slides were prepared by dropping the cells onto glass slides, digesting with $0.03 \%$ trypsin-EDTA solution and staining with Giemsa. (A) Typical G-banding metaphase was observed (magnification, $\mathrm{x} 400$ ). (B) G-banding karyotype was drawn based on G-banding patterns of chromosomes (original magnification, $\mathrm{x} 400$ ). (C) Chromosomes were amplified according to G-banding karyotype. The lengths of the straighter chromosome from each pair homologous chromosomes were measured and relative chromosome lengths were calculated.

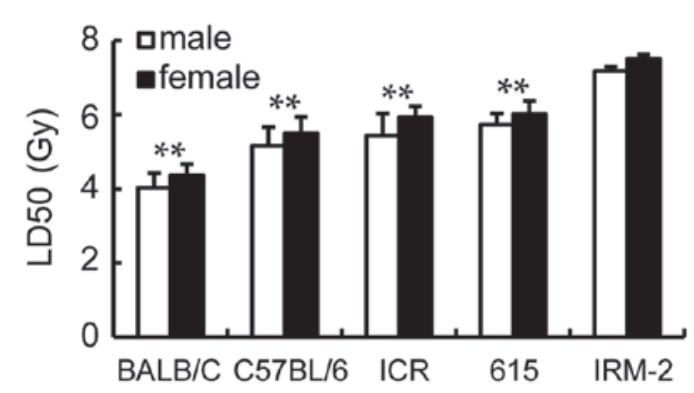

Figure 6. $\mathrm{LD}_{50}$ of various mice strains irradiated with ${ }^{137} \mathrm{Cs} \gamma$-radiation. $\mathrm{BALB} / \mathrm{c}, \mathrm{C} 57 \mathrm{BL} / 6, \mathrm{ICR} / \mathrm{JCL}, 615$ and IRM-2 mice were exposed to total body irradiation of 0-10 Gy (absolute lethal dose) ${ }^{137} \mathrm{Cs} \gamma$-rays and the mortality of each group was recorded 30 days after irradiation to calculate the $\mathrm{LD}_{50}$ for the mice $(\mathrm{n}=10) .{ }^{* *} \mathrm{P}<0.01$ vs. IRM-2 mice. $\mathrm{LD}$, lethal dose.

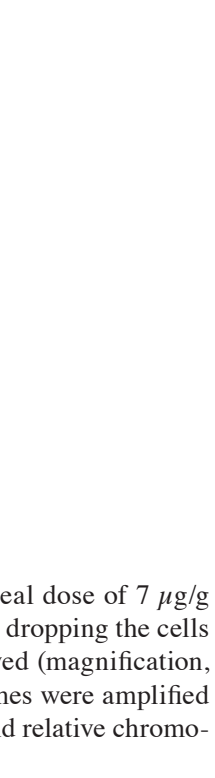



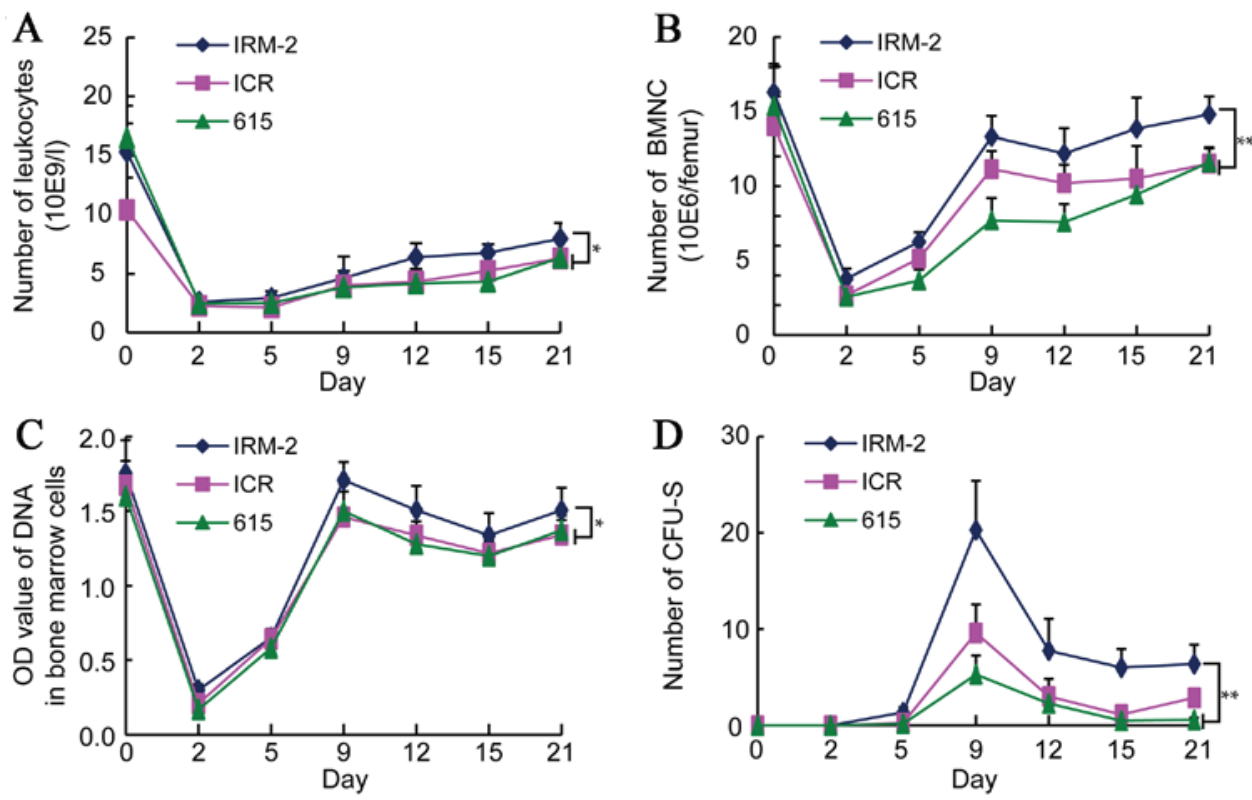

Figure 8. Changes in the number of leukocytes and in the hematopoietic function of mice irradiated with $\gamma$-radiation. The function of the hematopoietic system was examined to test the radiosensitivity of IRM-2 mice. IRM-2, ICR/JCL and 615 mice were irradiated with 4 Gy $\gamma$-radiation (n=8). (A) Whole blood was extracted from the orbital sinuses on 2,5,9,15 and 21 days after irradiation and the leukocytes were counted. "P $<0.05$ vs. IRM- 2 mice 12 to 21 days after radiation. (B) Whole blood was extracted from the orbital sinuses on 2, 5,9,15 and 21 days after irradiation and the BMNCs were counted. (C) BMCs were flushed from mouse femurs on 2, 5, 9, 15 and 21 days after irradiation and treated with a $0.2 \mathrm{~mol} / 1 \mathrm{HClO}_{4}$ solution. The DNA content of the BMCs was measured at $286 \mathrm{~nm}$. (D) Spleens were harvested from the abdominal cavity of mice on 2, 5, 9, 15 and 21 days after irradiation and CFU-S was counted under a microscope. "P<0.05, ${ }^{* *} \mathrm{P}<0.01$ vs. IRM-2 mice 9 to 21 days after radiation. BMNCs, bone marrow nucleated cells; BMCs, bone marrow cells; CFU-S, colony-forming unit-spleen.

CFU-S counts of the IRM-2 mice were higher than those of the ICR/JCL and 615 mice 9-21 days after irradiation $(\mathrm{P}=0.0004$ vs. ICR/JCL mice and $\mathrm{P}<0.0001$ vs. 615 mice; Fig. 8D), indicating that the extramedullary hematopoietic system had a protective effect against radiation exposure in the IRM-2 mice.

\section{Discussion}

As one of the most instructive experimental animals, inbred mice have been used in a wide range of research fields. In the present study, a novel inbred mouse strain, IRM-2, was generated by crossing ICR/JCL mice (maternal strain) with 615 mice (paternal strain). At the time of writing, the IRM-2 mouse is at generation F88 and has therefore met the inbred-strain requirement of having been bred for $>20$ generations. To determine the quality of a new animal strain, its basic biological characteristics have to be known. The IRM-2 inbred mice exhibited good traits: Strong reproductive capacity, stable physiological and biochemical indices and possessing few differences between individuals, thereby meeting the basic requirements of experimental animals.

Routine maintenance of experimental animals may ensure that valuable animal resources change as little as possible over time, thus ensuring that the biological research performed with experimental animals is as accurate and reproducible as possible (13). Although inbred mice genes are genetically stable, $5 \%$ of the original heterozygosity remains present even after 20 generations of inbreeding (14). Spontaneous mutations may occur during a long-term breeding process, possibly altering the biological characteristics of inbred mice (15). Therefore, to ensure that the IRM-2 mice remain well genetically characterized, a genetic monitoring program is necessary.

A coat-color gene test and a biochemical marker test were used to identify the genetic homogeneity of IRM-2 mice. The coat color of mice is genetically controlled by four locus alleles: a, b, c and d, which are located on chromosomes 2, 4, 7 and 9 respectively. In the majority of cases, there were four coat color phenotypes: AABBCCDD (wild-type), aabbCCDD (brown), aaBBCCDD (black) and AAbbCCDD (cinnamon). The coat color of the IRM-2 mice remained a constant cinnamon color during the breeding program. The following two features of biochemical markers are characteristic of inbred animals: i) Encoding biochemical marker genes are homozygous without any heterozygosity and ii) marker genes are highly consistent, with no difference between individuals. None of the biochemical markers tested from the IRM-2 mice at generations F23 and F38 exhibited polymorphisms or mutations. Collectively, the genetic monitoring data suggest that the IRM-2 mice were genetically homozygous and consistent.

The purpose of the present study was to generate a radioresistant animal model. To test the radiosensitivity of the resulting IRM-2 mice, the function of bone marrow was examined. Bone marrow, the hematopoietic system, is particularly sensitive to IR $(16,17)$. Once bone marrow is exposed to radiation-induced injury, the body produces symptoms, including hemorrhage, infection or anemia, that can be life-threatening. The parameters of BMNCs and the DNA content of bone marrow may be regarded as quantitative indices of radiation-induced injury to bone marrow and its recovery. BMNCs include granulocytes, megakaryocytes, lymphocytes and monocytes. The DNA content of BMCs reflects the proliferation of BMNCs. 
In the present study, the values of the number of BMNCs and DNA content of bone marrow progressively decreased in the early phase of radiation injury; in the recovery phase (2-21 days), they increased to their normal level. The increase in the DNA content of BMCs implied that proliferation activity of BMNC was increased. The tolerance of cells to radiation is increased by enhancing the ability of cells to repair DNA damage and the ability to promote the body's hematopoietic function, which aids the recovery and regeneration of BMNC and peripheral blood leukocytes (18). The number of BMNCs and the DNA content of the bone marrow from the IRM-2 mice were significantly higher than those of the 615 and ICR/JCL mice at different time points following irradiation, which suggested that the repair function of the bone marrow from the IRM-2 mice treated with radiation was robust.

When the hematopoietic microenvironment is severely damaged following exposure to high doses of radiation, a compensatory protective mechanism is induced; extramedullary hematopoiesis. Residual hematopoietic stem cells can migrate into the spleen to proliferate and spleen colonies develop in a process termed CFU-S. Due to the fact that this process enables self-renewal and multiple cell differentiation, CFU-S reflects the function of hematopoietic stem cells. The results of the present study demonstrated that the CFU-S counts of the IRM-2 mice after exposure to 4 Gy radiation were significantly higher than those of the parent mice. Collectively, IRM-2 mice exhibit high resistance to radiation due to bone marrow hematopoiesis and extramedullary hematopoiesis.

The dose of a substance that results in death in $\mathrm{P} \%$ of a test population is termed the $\mathrm{LD}_{\mathrm{P}}$. In radiation research field, the relevant $\mathrm{LD}_{\mathrm{P}}$ is the radiation dose that is lethal to $50 \%$ of test subjects in a designated period: Lethal dose 50 or $\mathrm{LD}_{50}$. When comparing $\mathrm{LD}_{50}$ between two test animals, the DRF is the common parameter of interest. In the present investigation, $\mathrm{LD}_{50}$ of the IRM-2 mice increased to $7.17 \mathrm{~Gy}$ (male) and 7.5 Gy (female), which were significantly higher than those of the parents, the 615 mice and ICR/JCL mice, giving a dose DRF value of 1.39 (male) and 1.37 (female). These data indicated that the IRM-2 mice had developed resistance to ionizing irradiation.

In the present study, the basic biological characteristics and the radiosensitivity of the IRM-2 mice were determined. Further systematic and comprehensive characterizations are required. To date, inbred IRM-2 mice have been used in studies of the biological effects of radiation, anticancer drug screening and nuclear medicine imaging research (19-23). The novel inbred strain characterized in the current study potentially constitutes a valuable mouse model for the study of radioresistance.

\section{Acknowledgements}

The present study was supported by the Special Foundation of the Ministry of Health (grant no. 201002009), the National Natural Science Foundation of China (grant nos. 30870583, 31170804, 31240052, 31200634 and 31300695), the Natural Science Foundation of Tianjin (grant nos. 09JCYBJC09300, 11ZCGYSY02400, 12JCYBJC15300, 12JCYBJC32900, 13JCYBJC23500 and 13JCQNJC11600) and the PUMC Youth Fund and Fundamental Research Funds for the Central Universities (grant nos. 2012G01 and 2012J05).

\section{References}

1. Steuber-Buchberger P, Wurst W and Kühn R: Simultaneous Cre-mediated conditional knockdown of two genes in mice. Genesis 46: 144-151, 2008.

2. $\mathrm{Yu} \mathrm{J}$ and McMahon AP: Reproducible and inducible knockdown of gene expression in mice. Genesis 44: 252-261, 2006.

3. Berndt A, Sundberg BA, Silva KA, Kennedy VE, Richardson MA, Li Q, Bronson RT, Uitto J and Sundberg JP: Phenotypic characterization of the KK/HIJ inbred mouse strain. Vet Pathol 51: 846-857, 2014.

4. Serpi R, Klein-Rodewald T, Calzada-Wack J, Neff F, Schuster T, Gailus-Durner V, Fuchs H, Poutanen M, Hrabrè de Angelis M and Esposito I: Inbred wild type mouse strains have distinct spontaneous morphological phenotypes. Histol Histopathol 28: 79-88, 2013.

5. Barden EK, Rellinger EA, Ortmann AJ and Ohlemiller KK: Inheritance patterns of noise vulnerability and 'protectability' in (C57BL/6J x CBA/J) F1 hybrid mice. J Am Acad Audiol 23: 332-340, 2012.

6. Hutsell BA and Newland MC: A quantitative analysis of the effects of qualitatively different reinforcers on fixed ratio responding in inbred strains of mice. Neurobio Learn Mem 101: 85-93, 2013.

7. Kilikevicius A, Venckunas T,Zelniene R, Carroll AM,Lionikaite S, Ratkevicius A and Lionikas A: Divergent physiological characteristics and responses to endurance training among inbred mouse strains. Scand J Med Sci Sports 23: 657-668, 2013.

8. Chesler EJ, Plitt A, Fisher D, Hurd B, Lederle L, Bubier JA, Kiselycznyk C and Holmes A: Quantitative trait loci for sensitivity to ethanol intoxication in a C57BL/6J×129S1/SvImJ inbred mouse cross. Mamm Genome 23: 305-321, 2012.

9. Perez CJ, Dumas A, Vallières L, Guénet JL and Benavides F: Several classical mouse inbred strains, including DBA/2, $\mathrm{NOD} / \mathrm{Lt}, \mathrm{FVB} / \mathrm{N}$, and SJL/J, carry a putative loss-of-function allele of Gpr84. J Hered 104: 565-571, 2013.

10. Meng A, Wang Y, Brown SA, Van Zant G and Zhou D: Ionizing radiation and busulfan inhibit murine bone marrow cell hematopoietic function via apoptosis-dependent and-independent mechanisms. Exp Hematol 31: 1348-1356, 2003.

11. Hongying W, Yueying W, Lu L, Junling Z, Deguan L and Aimin M: Comparison of the radiation sensitivity in vitro of bone marrow cells from three mouse strains. Chin J Compar Med 19: 56-58, 2009.

12. Junxu L, YueHua C, Wang Y, Feng X, Wang L, Rong-Zhen S, Desen L and Fuying L: Detection of biochemical markers in inbred MIJ and HFJ rats. Acta Lab Anim Sci Sin 19: 428-430, 2011.

13. Taft RA, Davisson M and Wiles MV: Know thy mouse. Trends Genet 22: 649-653, 2006.

14. Yuan R, Flurkey K, Meng Q, Astle MC and Harrison DE: Genetic regulation of life span, metabolism, and body weight in pohn, a new wild-derived mouse strain. J Gerontol A Biol Sci Med Sci 68: 27-35, 2013.

15. Fahey JR, Katoh H, Malcolm R and Perez AV: The case for genetic monitoring of mice and rats used in biomedical research. Mamm Genome 24: 89-94, 2013.

16. Chen J: Animal models for acquired bone marrow failure syndromes. Clin Med Res 3: 102-108, 2005.

17. Jagetia GC and Venkatesh P: Inhibition of radiation-induced clastogenicity by Aegle marmelos (L.) correa in mice bone marrow exposed to different doses of gamma-radiation. Hum Exp Toxicol 26: 111-124, 2007.

18. Cuixia Z, Xuefen Y, Li S, Xiaodan W and Tao X: Protective Effects of radaway radioprotection capsule on radiation injured mice. Chin J Radiol Health 21: P259-260, 2012.

19. Wang YY, Zhou ZW, Shen X, et al: Radiation-protective effect of E838 on IRM-2 and ICR/JCL mice. Chin J Compar Med 14: 332-335, 2004.

20. Yueying W, Ruqin W and Zhong-Ping Z: Radiation protective effects of four kind of estrogens on mice. Chin J Radiol Med Prot 25: 39-40, 2005.

21. Li L, Peizhen H, Yujun Y, Yueying W, Junqi W and Meijia L: Application of ${ }^{99} \mathrm{Tc}^{\mathrm{m}}$-HL91 in the hypoxia study of malignant lymphoma. Chin J Nucl Med 22: p118-119, 2002.

22. Zhou ZW, Liu PX, Li SN, et al: Inhibiting of 9002 on the growth of xenografted tumor of IRM-2 mice. Chin J Lab Anim Sci 12: 173,2002

23. Jingying $Y$, Qing W, Guofan L, Chuanjie M, Peizhen H and Xuemin F: An experimental study of inflammation imaging with '99Tcm-HYNIC-fMLFK' in IRM-2 inbred mice. Chin J Nucl Med 23: p179-181, 2003. 